\title{
SAXO, the SPHERE extreme AO system On-sky final performance and future improvements
}

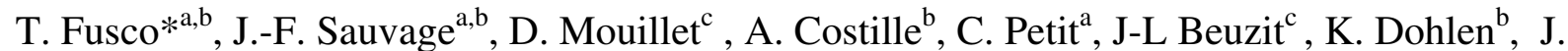

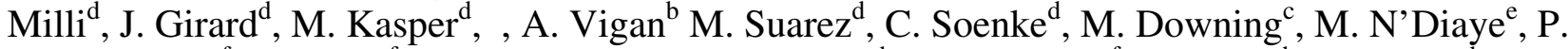

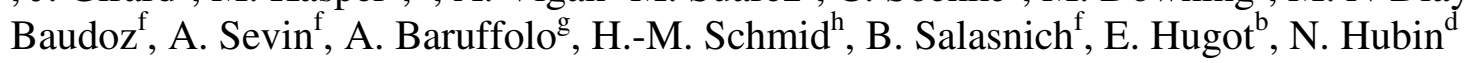 \\ ${ }^{a}$ ONERA, 29 avenue de la Division Leclerc, 92322 Châtillon, France \\ ${ }^{\mathrm{b}}$ Aix Marseille Université, CNRS, LAM UMR 7326, 13388, Marseille, France \\ ${ }^{c}$ IPAG (UMR 5274) BP 53. F-38041 Grenoble, France \\ ${ }^{\mathrm{d}}$ ESO Karl-Schwarzschild-Str. 285748 Garching bei München, Germany \\ ${ }^{\mathrm{e}}$ Space Telescope Science Institute - STScI - 3700 San Martin Drive, Baltimore MD 21218 USA \\ ${ }^{\mathrm{f}}$ LESIA Observatoire de Paris, 5, place Jules Janssen 92195 Meudon, France \\ ${ }^{g}$ INAF Osservatorio Astronomico di Bologna ,Via Ranzani 1, 40127, Bologna, Italy \\ ${ }^{\mathrm{h}}$ ETH, Rämistrasse 101, 8092 Zurich, Switzerland
}

\begin{abstract}
The SPHERE (Spectro-Polarimetric High-contrast Exoplanet Research) instrument aims at detecting extremely faint sources (giant extrasolar planets) in the vicinity of bright stars ${ }^{1}$. Such a challenging goal requires the use of a very-highorder performance Adaptive Optics [AO] system feeding the scientific instruments with a quasi-perfect flat wave front corrected from all the atmospheric turbulence and internal defects. This AO system, called SAXO (Sphere Ao for eXoplanet Observation) is the heart of the instrument, a heart beating 1200 time per second and providing unprecedented image quality for a large ground based telescope at optical/near infrared wavelength. We will present the latest results obtained on-sky, demonstrating its exceptional performance (in terms of correction quality, stability and robustness) and tremendous potentiality for high contrast imaging and more specifically for exoplanet discovery.
\end{abstract}

Keywords: Instrumentation: Adaptive Optics, Coronagraph, High Contrast Imaging

\section{INTRODUCTION}

Direct detection and spectral characterization of exoplanets is one of the most exciting but also one of the most challenging areas in the current astronomy. In that framework, the SPHERE (Spectro-Polarimetry High-contrast Exoplanet Research) instrument has been recently installed on UT3, one of the four 8-m telescopes of the European Southern Observatory Very Large Telescope (ESO VLT) at Paranal (Chile). The main scientific objective of SPHERE [1] is the direct detection of photons coming from giant extrasolar planets (between 1 and 20 Jupiter masses). Any detection will then be followed by a first characterization of the planet atmosphere (clouds, dust content, methane, water absorption...). In addition, the survey of an extended number of stars (typically a few hundreds) is mandatory for performing meaningful statistical studies. Such extremely challenging scientific objectives directly translate into a relatively complex high-contrast instrument. Coronagraphic and smart imaging capabilities are essential for reaching the high contrast (close to the optical axis) required for direct extrasolar planet detection. From the ground, the core of any high-contrast instrument is an extreme adaptive optics (XAO) system. Such a system must be capable of making corrections for the perturbations induced by the atmospheric turbulence as well as for the internal aberrations of the instrument itself. In that context, SAXO (Sphere Ao for eXoplanet Observation), the XAO system of SPHERE has been designed, integrated, tested in labs and finally on sky, by a European wide group of AO scientists and Engineer, led by ONERA with the strong support of ESO. A comprehensive design of SAXO and the detailed description of the main system choices can be found in [2]. The final European AIT (Assembling Integration and Test) of SAXO have taken place in Grenoble from 2012 to 2013 [3]. We report here the final SAXO performance obtained during the four commissioning runs (obtained from May to November 2014) on UT3 one of the four eight meter class telescope of the VLT, as well as the first year of operation (2015 and beginning of 2016), 


\section{SPHERE IN A NUTSHELL}

The performance of AO [2, 3,4] of an exoplanet hunter and its impact on the final detectivity is of prime importance: a better AO correction leads to a better coronagraph extinction and less residual (quasi static or fast varying) defects. To meet the requirements in terms of detection the proposed design of SPHERE is divided into four subsystems, namely, the common path optics and three science channels. The common path includes pupil-stabilizing fore-optics (tip-tilt and derotator) where insertable polarimetric half-wave plates are also provided, SAXO the XAO system with a visible wavefront sensor, and near infrared (NIR) coronagraphic devices in order to feed the infrared dual-imaging spectrograph (IRDIS) [5] and the integral field spectrograph (IFS) [6] with a highly stable coronagraphic image in the NIR. The third channel is dedicated to visible differential polarimetry (ZIMPOL) [7].

The three scientific channels gather complementary instrumentation to maximize the probability of exoplanet detection and to give us access to a large range of wavelengths and information (e.g., imaging, spectra, and polarization ${ }^{7}$.

The concept behind this very challenging instrument is illustrated in Figure 1. Since May 2014, SPHERE is installed on the third unit telescope (Melipal) of the VLT. After 4 months of extensive and comprehensive tests (robustness, performance, easiness of use). It is now open to the astronomical community for the next observation period (April 2015). After less than one year of operation, the scientific returns of SPHERE is already quite exceptional with more the 20 referee papers on several topics (disk, solar system bodies, exoplanet ...)

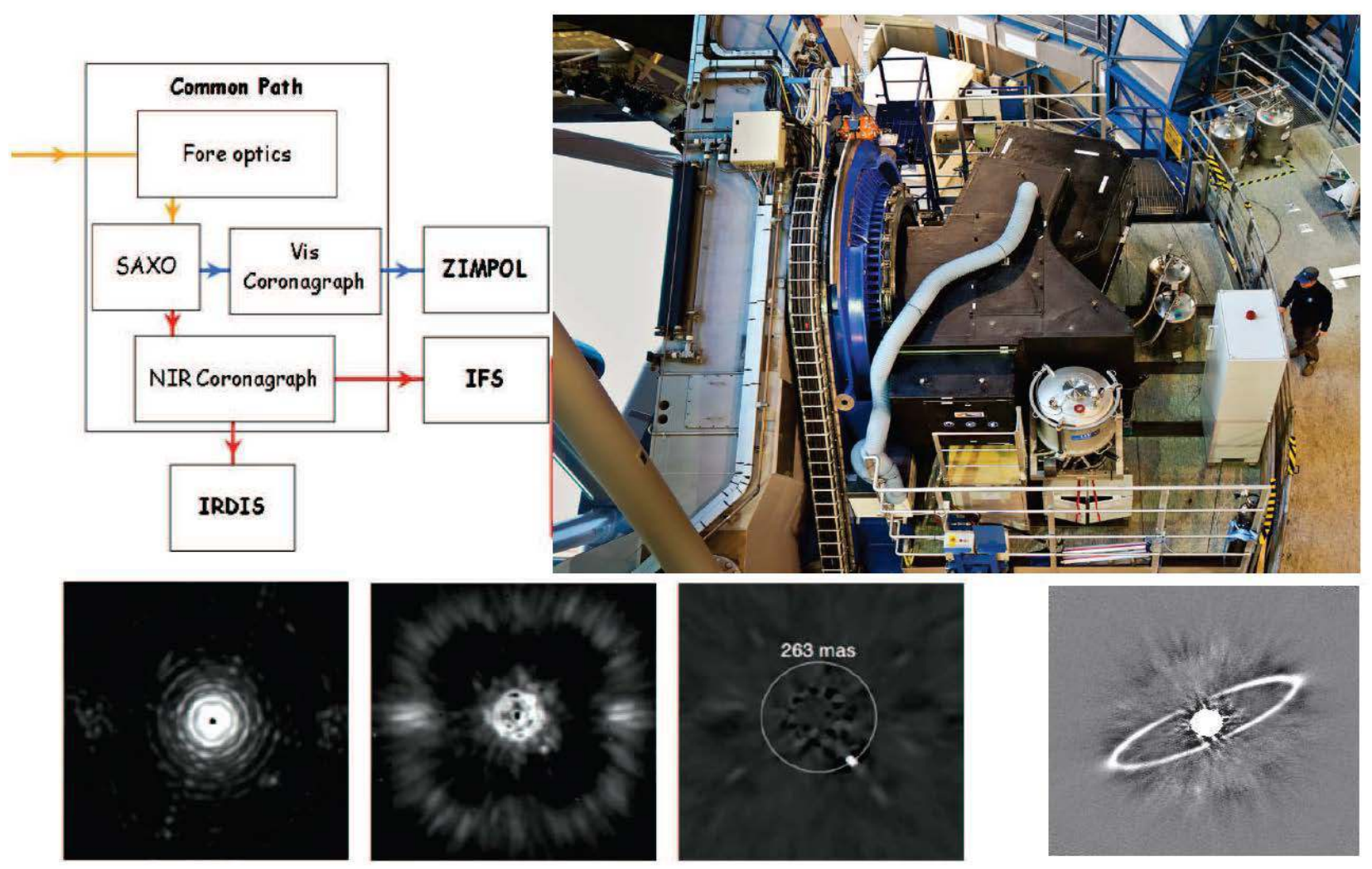

Figure 1 [up-left] Global concept of the SPHERE instrument, indicating the four subsystems and the main functionalities within the common path subsystem. Optical beams are indicated in red for NIR, blue for Vis, and orange for common-path. [up-right] SPHERE on the telescope: $6 \times 4 \times 2.5 \mathrm{~m}^{3}, 8$ tons, resting on 3 anti-vibrations pillars. A monster with unprecedented image sharpness and optical stability. [down-left] As an example: J-band saturated image, coronagraphic and processed images with a companion, iota Sgr b, a very low-mass star 4,000 times fainter at 0.24 " from its parent star. [down-right]: dust ring around the star HR 4796A 


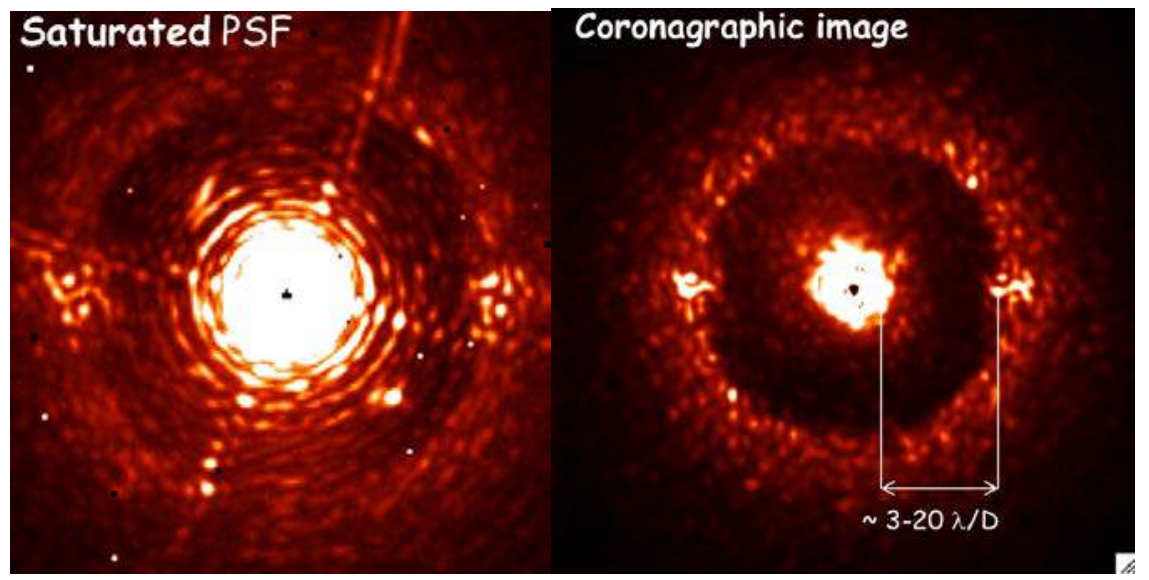

Figure $2 \mathrm{~J}$ band classical and coronagraphic PSF. The classical PSF is saturated in order to show the corrected area and the multiple Airy rings. The coronagraphic image shows a very nice darl halo produced by the AO up the DM cut of frequency $(\lambda / 2 \mathrm{~d}$, d being the interactuator spacing)

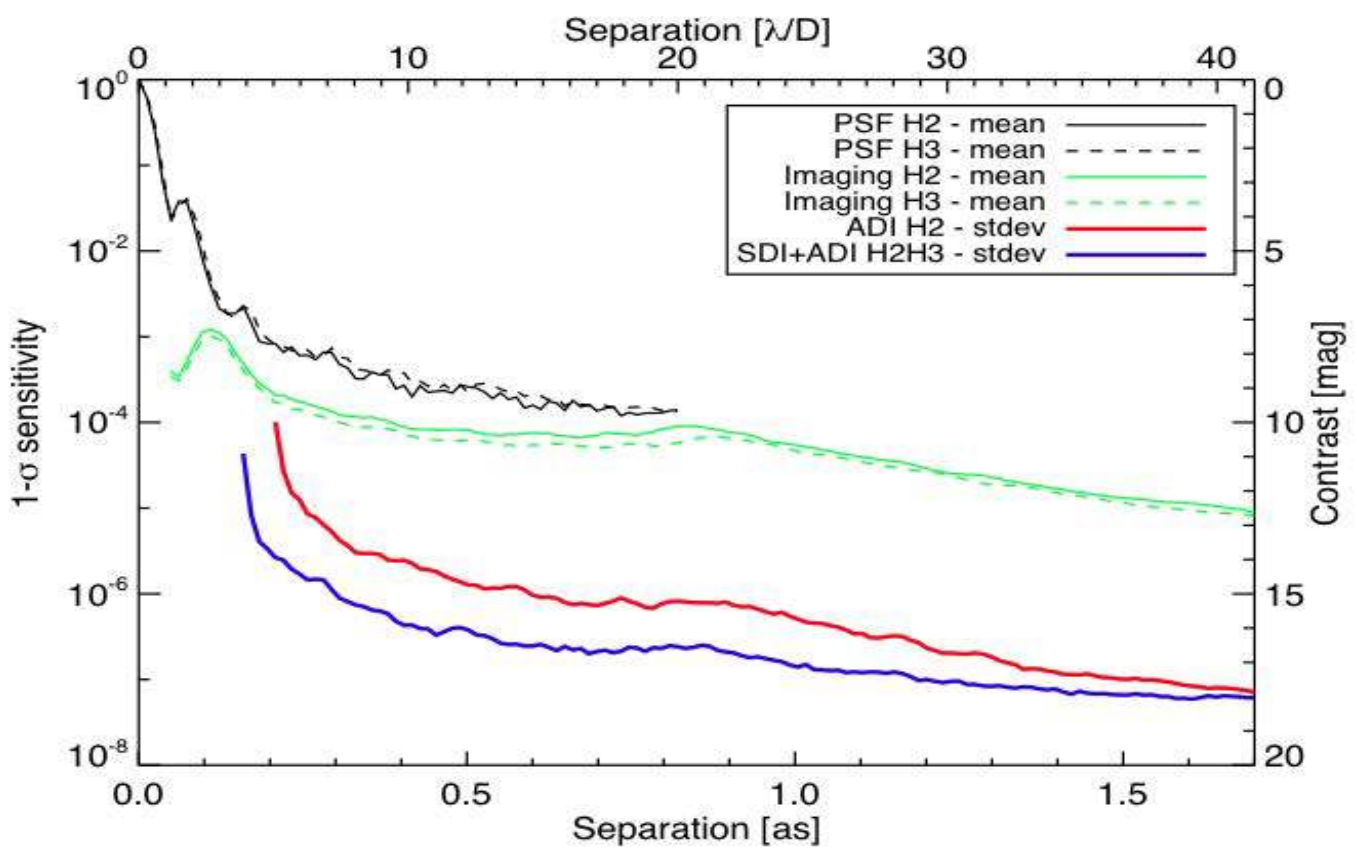

Figure 3 SPHERE typical contrast curve using on-sky data. The combination of extreme AO, efficient coronagraph and clever differential technics (ADI and SDI) allows obtaining contrast better to $10^{-6}$ down to less then $0.3^{\text {', }}$

\section{SAXO, THE SPHERE EXTREME AO SYSTEM}

SAXO measures and corrects any wave-front perturbation (rapidly varying turbulence or quasi-static instrumental speckles [8]) in order to ensure an unprecedented image quality on ground -based telescope. It gathers some of the most worldwide advanced components and AO concepts. It incorporates a fast (800 Hz bandwidth) tip-tilt mirror, [TTM] an active Toric mirror[9] and a 41x41 actuators (1377 active) deformable mirror [DM]. Wave-front sensing is based on a filtered Shack-Hartman concept, using state of the art EMCCD[10] running up to $1200 \mathrm{~Hz}$ with less than 0.1 e- of equivalent read-out-noise. The filtering pinhole designed for removing aliasing effect $[11,12]$ is automatically adjusted as 
a function of the atmospheric conditions. The unprecedented EMCCD characteristics combined with clever centroiding technics (weighted center of gravity[13,14]) allows SAXO to have impressive limit magnitudes:

- the ultimate performance limit magnitude for which the instrument meets its initial requirements in terms of wave front correction, $\operatorname{magR}=9-10$, where the SR In H band is higher or equal to $90 \%$

- the "classical" limit magnitude for which the AO system still provide a significant gain (typically a factor 5 to 10 w.r.t. the purely turbulent case), $\operatorname{magR}=15-16$.

A real time computer (SPARTA) controls the TTM and DM with a final latency of $80 \mu$ s. It incorporates a modal gain optimization for DM modes and a Linear-Quadratic-Gaussian law[15] specifically designed for TTM in order to automatically identify and filtered out up to 10 vibration peaks randomly spread between 10 and $300 \mathrm{~Hz}$. This last feature allows reaching a final residual jitter of less than 2 mas rms (1/20 of the H-band diffraction) which is fundamental to ensure the optimal operation of the various SPHERE coronagraphs. SAXO has also two auxiliary loops which ensure

- a fine centering (at $1 \mathrm{~Hz}$ ) on the coronagraph by compensating for chromatic effects using a specific IR tip-tilt sensor [16] and a Differential Tip Tilt mirror;

- A pupil centering (at $0.1 \mathrm{~Hz}$ ) to compensate for telescope run-out. It is based on the analysis of SH edge subapertures flux and the correction using a small tip tilt mirror located very close to a focal plane [17].

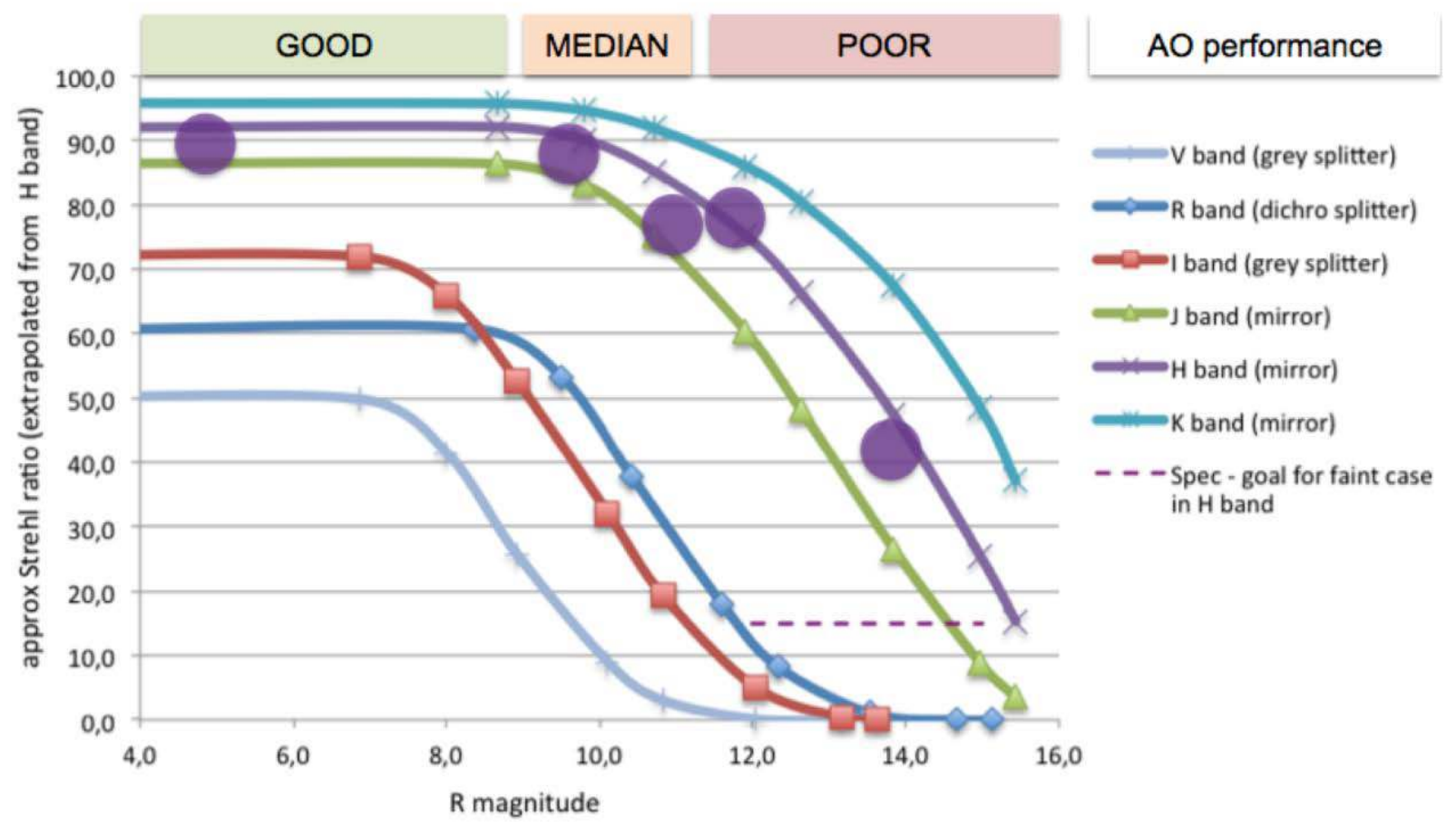

Figure 4 SAXO performance measured in labs (various curves) and on sky (dots).

All these features put together allows providing SPHERE with an unprecedented flat wave-front corresponding to Strehl Ratio larger than $90 \%$ in $\mathrm{H}$ and diffraction limited images at visible wavelength (I and R). In addition to its very high performance, SAXO has been designed and developed to be as automatic as possible. It means that the system autooptimizes itself using its own internal data (without any user intervention). In the same spirit, calibration and verifications (quality checks) processes are completely automatic. Therefore SAXO, and more generally SPHERE can be operated by a non-specialist without compromising on performance. 


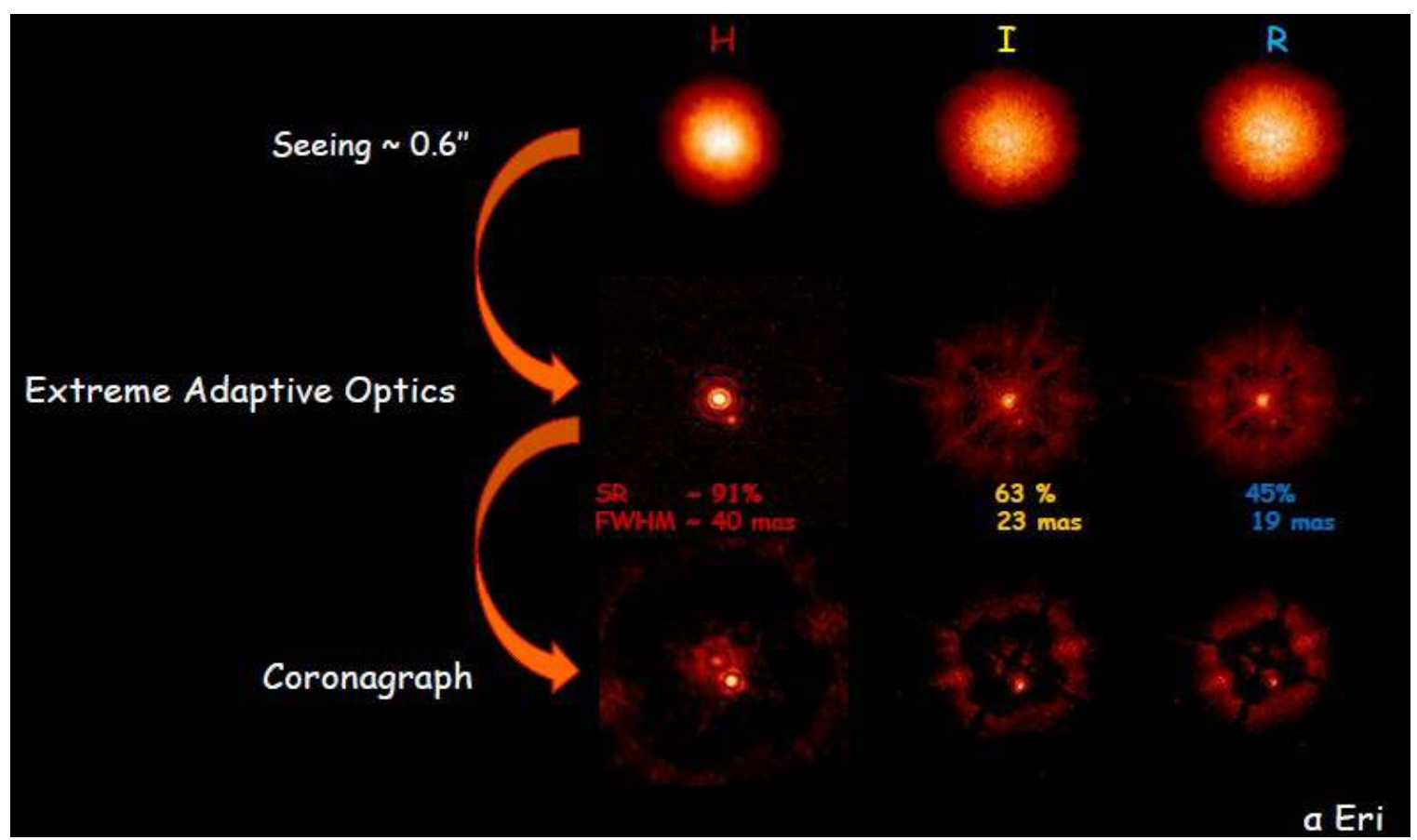

Figure 5 NIR(H) and VIS(I and R) imaging of the same object. All the images have the same FoV (1.8 arcsec).

From top to bottom: seeing limited image (seeing is estimated to 0.6" using RTC data), AO corrected images and coronagraphic images. The latest ones clearly reveal a low mass companion. Diffraction limit regime is fully reached in

$\mathrm{H}$ (40 mas) and I (23 mas) and 19 mas of FWHM has been achieved in R (diffraction limit in R: 18 mas). This corresponds to a world record for monolithic telescope either on the ground or in space.

\section{SOME SAXO SPECIFIC FEATURES}

\subsection{Ultimate performance on internal source}

Let us first consider the ultimate performance of the SPHERE system itself. The source is put at the entrance focal plane: FP1. SAXO loops are closed, Non-Common Path Aberrations (NCAP) have been measured using a Phase diversity algorithm and pre-compensated for by modifying the WFS reference slopes [8].

Considering only the correction of the WFS path aberrations (by using an internal fiber at the entrance of the WFS arms), the SPHERE optical quality is already impressive with a SR in $\mathrm{H}$ around $97.5 \%$ which corresponds to $41 \mathrm{~nm}$ rms of residual aberrations (note that the overall budget for IRDIS optics between dichroic and detector is $36 \mathrm{~nm}$ rms which is in very good agreement with our measurements). This good result is due to

1. the exceptional quality of the SPHERE optics (all of them have been specified and delivered with less than a few $\mathrm{nm}$ rms) with specific attention to the non-common ones.

2. the overall system alignment (and the days and weeks spent to make it both quasi perfect)

On the top of that, an optimized phase diversity algorithm has been applied to measure and correct for the very last residual aberrations. By doing that a SR of more than $99 \%$, that is $25 \mathrm{~nm}$ rms, is measured whereas the full iterative NCPA process of Phase Diversity measurements + reference slope modifications converges towards less than a few $\mathrm{nm}$ rms of residuals. The difference between the two numbers is essentially due to high spatial frequency aberrations which are neither sensed nor corrected by the NCPA loop but still presents in the image itself.

Nevertheless, achieving more than $99 \%$ of SR on an 8 tons instruments with a volume of $6 \mathrm{mx} 4 \mathrm{mx} 2.5 \mathrm{~m}$ is already quite a performance and by far exceed the original specifications. A detailed description of the various wavefront sensor strategies in SPHERE could be found in [4]. 


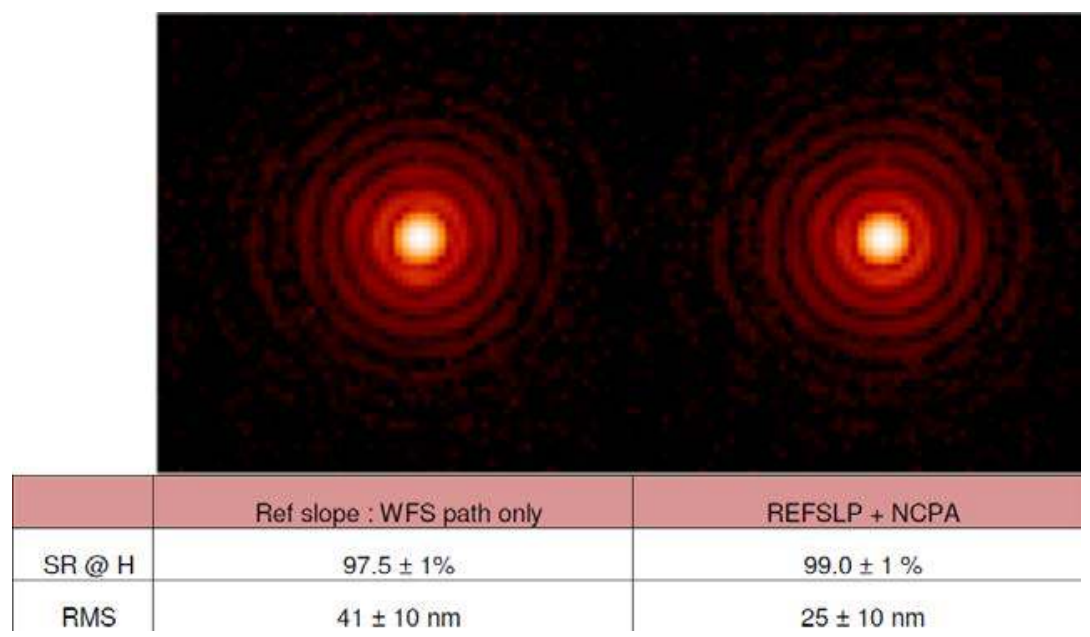

Figure 6 SPHERE internal performance without and with NCPA compensation. This was obtained during AIT period with a limited number of dead actuator (4 only). $99 \%$ of SR in $\mathrm{H}$ (or in other words less than $25 \mathrm{~nm}$ rms of residual aberrations) has been obtained at the very end.

\subsection{AO temporal behavior}

Temporal aspects are critical for XAO applications in order to improve the overall performance (better atmospheric correction as well as a better rejection of telescope and instrument vibrations). Nevertheless it requires significant effort both in terms of hardware (we need very fast component) and software (we need to be able to control them efficiently). In the SPHERE project, the two aspects have been carefully considered and several innovative solutions have been proposed. A detailed description of the SPHERE temporal behavior could be found in [15]. In a nutshell, the AO system has its expected temporal bandwidth of $70 \mathrm{~Hz}$ with a $1200 \mathrm{~Hz}$ frame rate and an $81 \mu$ s pure RTC latency.
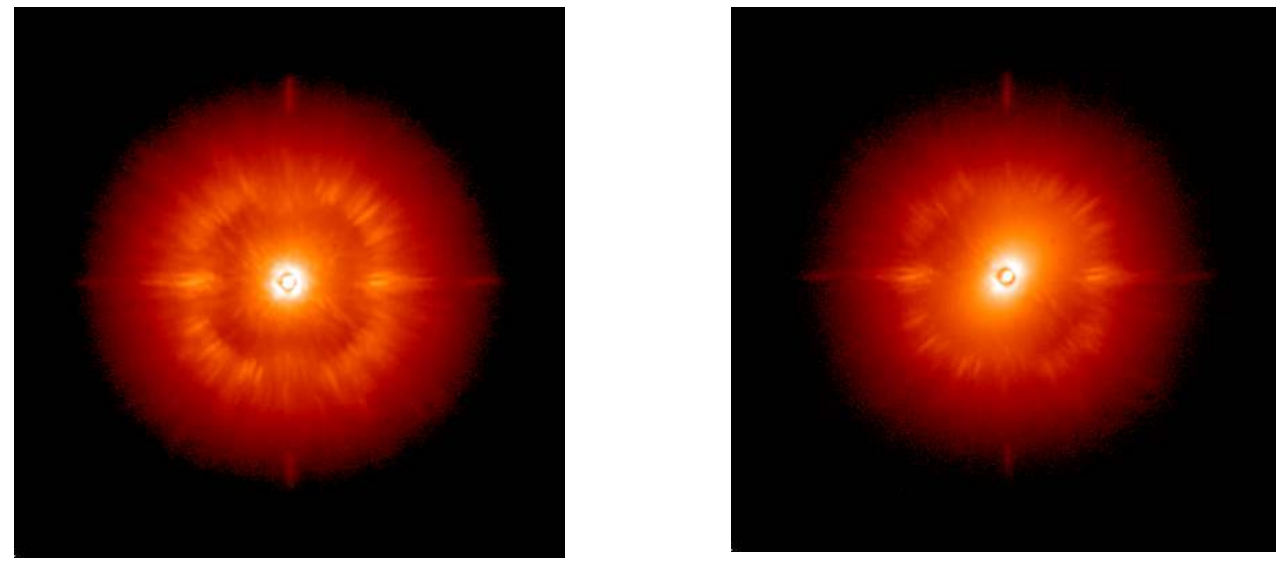

Figure 7 coronagraphic images under good $(<3 \mathrm{~m} / \mathrm{s})$ [left] and bad $(>20 \mathrm{~m} / \mathrm{s})$ [right] wind conditions. In both cases seeing conditions are around 0.9 ”. SR is estimated (using RTC data) to respectively 85 and $57 \% \pm 3 \%$ in $\mathrm{H}$ band.

The DM is controlled using a classical control law based on an optimal modal gain integrator (OMGI) scheme. The two specific Tip Tilt modes are treated separately. The strong requirements in terms of residual jitter led us to consider a more complex thus more efficient control law for these particular modes. A Linear Quadratic Gaussian approach has been considered. This predictive scheme allows to optimally dealing with the temporal evolution of the turbulent phase as well as with the unexpected vibrations produced either by the telescope or the instrument itself. Such defects have been clearly identified in many AO systems as one of the most critical aspect for achieving ultimate performance. In the 
SPHERE case, this point is even more essential since the residual jitter requirement at the coronagraphic mask level is extremely challenging (less than 3 mas rms). Therefore a specific on-line identification of vibration peaks has been developed and coupled with a LQG control law which will filter them out of the AO loop system even though their temporal frequency is above the AO system bandwidth itself. It is demonstrated on sky that only the use of an LQG aooroach allows reaching the residual jitter specification. It allows reducing the jitter rms by at least a factor 3 to 4 , going from typically 10 to 12 down to less than 3 mas rms (see Figure 8)!
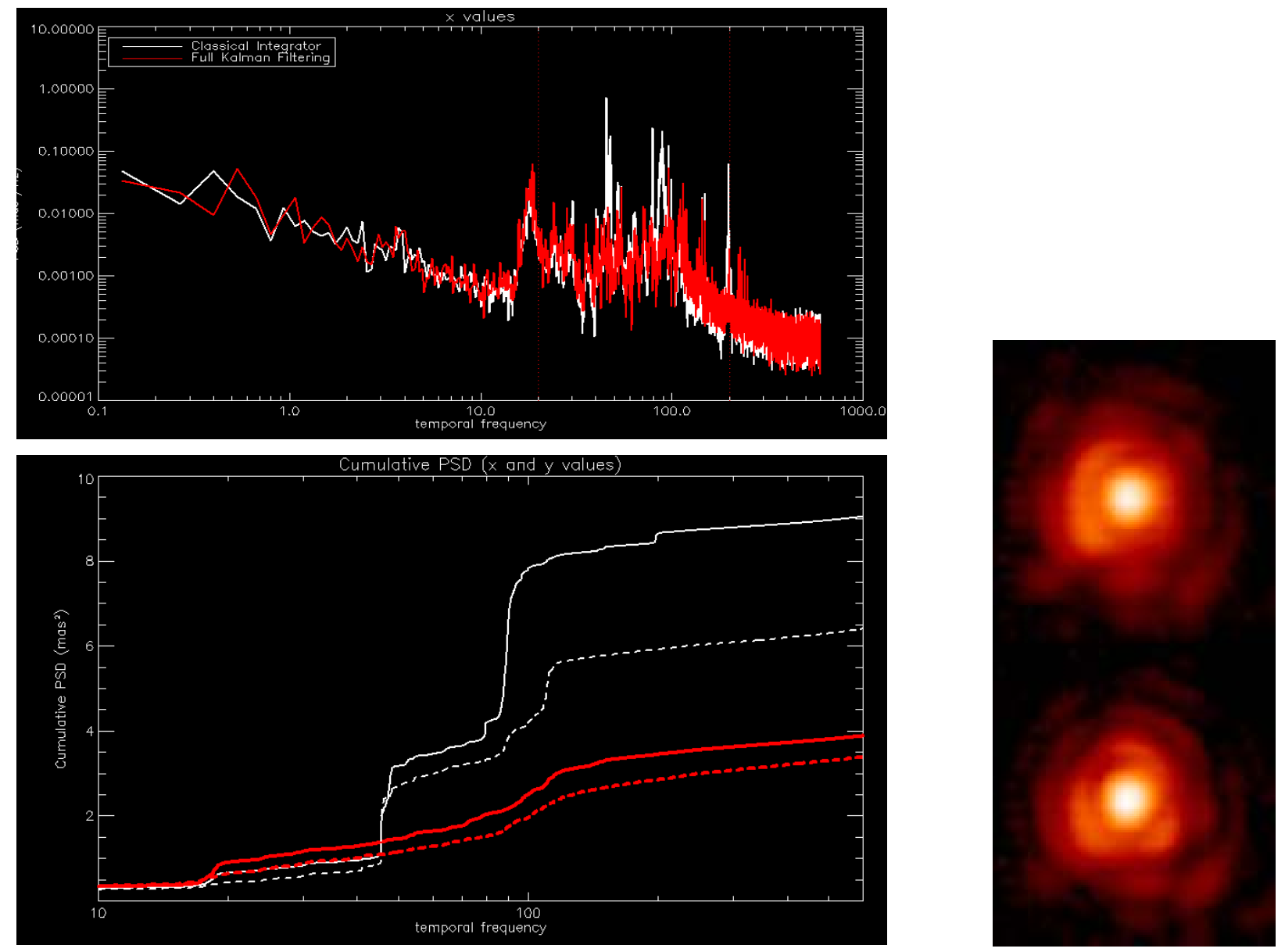

Figure 8 [Left] PSD and Cumulative PSD of both Tip and Tilt modes with [red] and without [black] LQG. The vibration filtering is clearly visible around 43 and $85 \mathrm{~Hz}$. The final residual jitter goes from $2.5 / 3$ mas per axis (in the classical integrator case) down to les than 2 mas per axis in the Kalman case. The corresponding gain in term of image quality [right] is shown on DTTS images (a SR gain of roughly $10 \%$ has been measured between classical and LQG data).

\subsection{Spatially filtered SH}

The goal of the spatially filtered SH is to remove the aliasing effects in the WFS measurements [11,12]. Figure 9 shows the very nice behavior of coronagraphic images (i.e. the increase of $\mathrm{AO}$ correction and ultimate performance) as a function of the spatial filter size. The gain brought by the spatially filtered SH is up to a factor 3. We have managed to go down to $1.1 \lambda / \mathrm{d}$ (d being the sub-aperture size) which is close to the theoretical limit and which allows us to have a real aliasing free WFS. A detailed analysis and comparison with simulation results can be found in [13]. The key point being that on-sky results are fully compatible with simulation ones and that the gain in terms of raw contrast due to the Filtering device is up to 3 (see Figure 9 and 7). The concept is now fully operational on SPHERE; the size of the spatial filter is automatically adjusted before each observation depending on seeing conditions. 


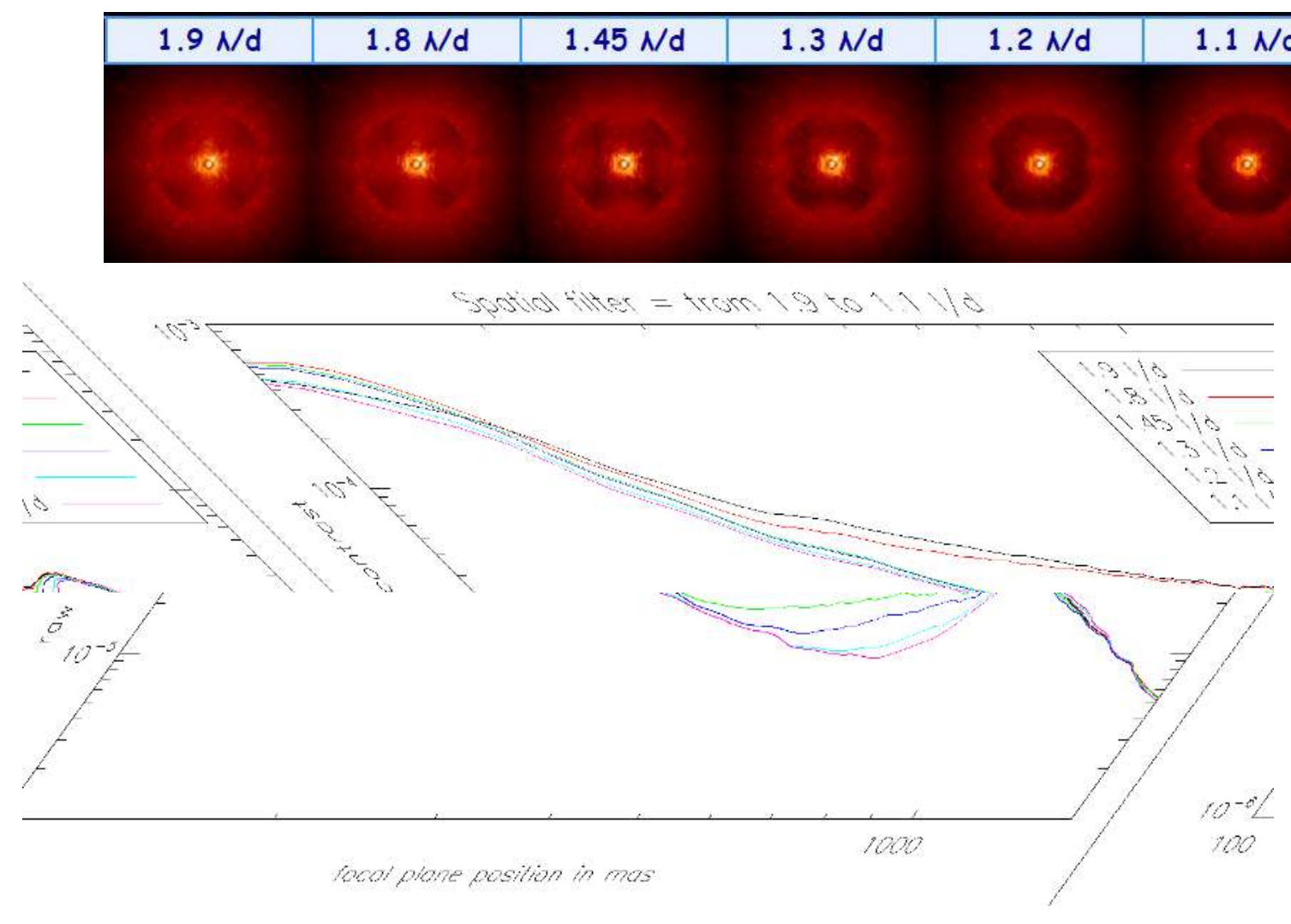

Figure 9 Evolution of the coronagraphic images as a function of the spatial filter size. Up: coronagraphic images, Down: circular profiles, normalized to peak

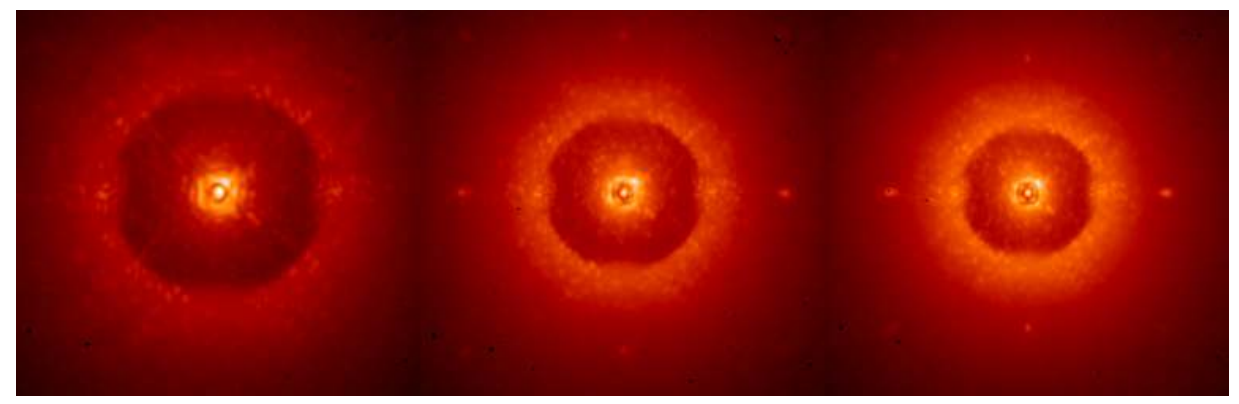

Figure 10 Coronagraphic images obtained on IRDIS in H, J and Y band (from left to right). Spatial filter size was 1.2 $\lambda / \mathrm{d}$. The "round" shape of the corrected area comes from the modal optimization of the AO loop. The last 300 modes (over 1377) are not controlled for stability reason.

\subsection{Operation in strong seeing conditions and on extended object}

SPHERE main WFS is a filtered SH with a fully adjustable pinhole. It allows us to work on extended object (larger than $2 \operatorname{arcsec}$ ) and under strong seeing conditions. We were able to close the loop and obtain a stable correction up to seeing larger than 1.5 arcsec.

These two features are demonstrated in Figure 11. The gentle degradation of AO performance w.r.t seeing is shown as well as the very beautiful results of a loop closed on Neptune. 

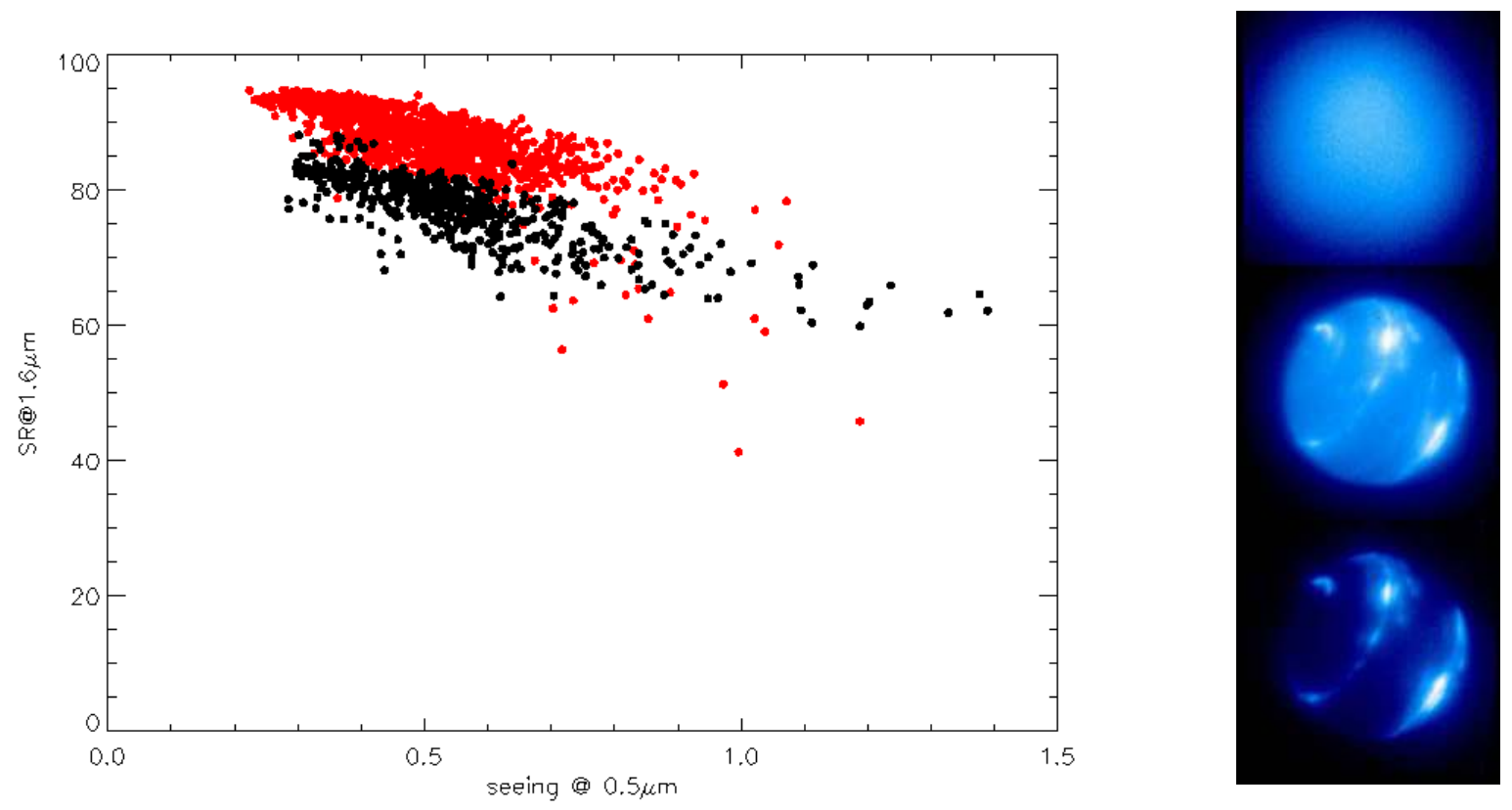

Figure 11 [Left] SPHERE performance as a function of seeing. In black, the data obtained during com1 before the system optimization and the correction of the telescope-M2 residual vibrations. In red, the data obtained during com 2 and 3 after system optimization. [Right] Neptune observerd under bad seeing condition ( 1.5') demonstrating the system capability to close the loop on extended object (>2") and in bad seeing conditions (from top to bottom : seeing limited image and $\mathrm{AO}$ corrected image in $\mathrm{H} 2$ and $\mathrm{H} 3$ band)

\subsection{VIS performance}

A unique feature of SPHERE is its visible imaging / coronagraphy / dual polarimetry capabilities. Thanks to the ZIMPOL instrument, we can have access to photons down to the blue light ( $\mathrm{V}$ band).

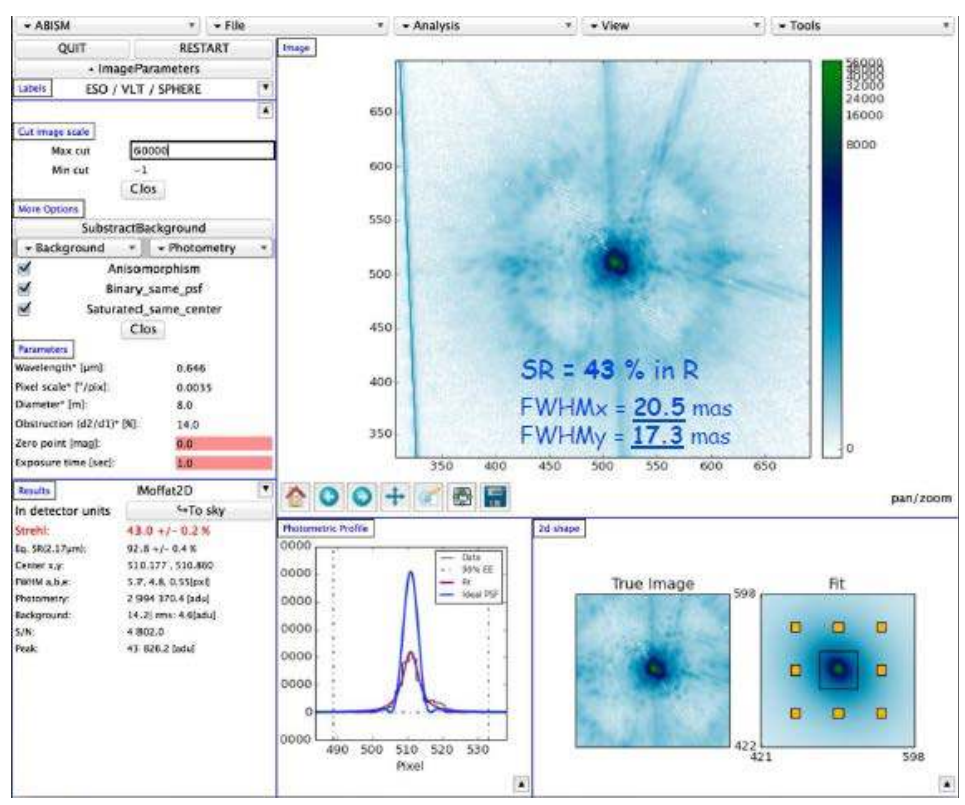

Figure 12 SR measurement on a R-band PSF. 45\% de SR is computed with a FWHM around 20mas (17.3 and 20.5 depending on the axis) 
The combination of the SAXO exceptional performance and an extremely good and stable camera allows us to obtain unprecedented images at very short wavelength on a 8-m class telescope. Strehl ratio of a few tens of percent is obtained with near limited diffraction images in R band (between 17 and 20 mas depending on the axis), the main limitation in that case being the residual jitter. This is, up to now, a world record for a monolithic telescope (both space- or groundbased).

\subsection{Low flux behavior}

Even though SPHERE has been primarily designed for high contrast on bright targets (nominal performance must be obtained up to V-mag $=9$ ), a very nice feature of the instrument is its capability to close the loop and obtain significant correction in $\mathrm{H}$ band even for relatively faint guide star.

This has been made possible thanks a unique combination of features: a photon noise limited EMCCD (RON $<0.2$ e- per pixel and per frame), an efficient weighted centroiding algorithm [14], an optimal modal gain integrator control law and an optimized optical train in terms of transmission and emissivity. Hence the limit magnitude of SPHERE is

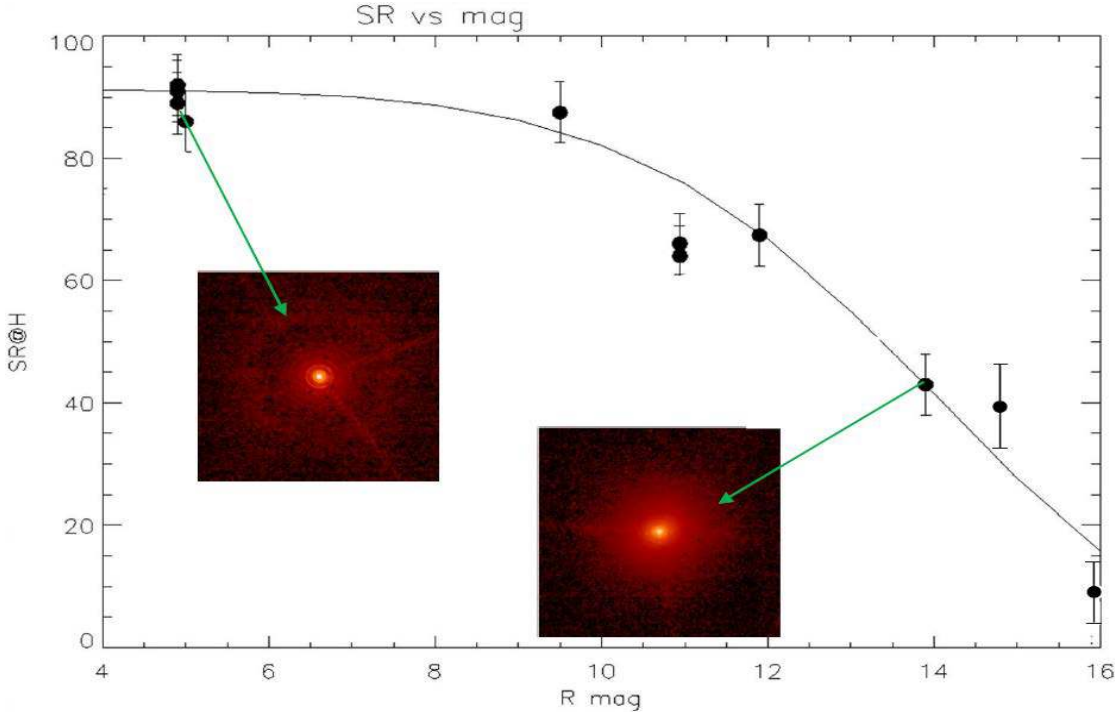

Figure 13 Highlight on 3 images: high flux, medium flux and low flux regimes. better than the initial specification.

Indeed, the nominal performance is obtained above magnitude 9 and we can observe a very gentle decrease of the SR as a function of the magnitude up to almost mag 16 (see Figure 14). Even though SPHERE has 8 times more sub-apertures than NACO (i.e. 8 times less photon per sub-aperture for the same magnitude), its performance outperforms NACO (in its natural visible guide star mode) ones on its whole magnitude range.

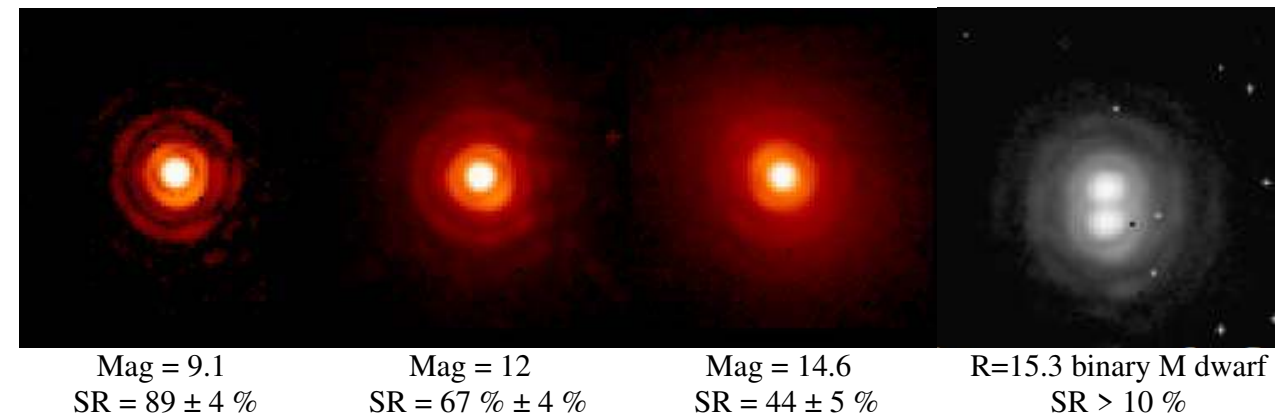

Figure 14 SR (H band) vs R-mag for various observations obtained during commissioning runs 2, 3 and 4 . The error bar is estimated as a function of the uncertainties on the system parameters (over sampling detector transfer function ...) and the quality of image reduction (background subtraction, bad pixel removal etc..). On the very tight right a very faint ( magR $=15.3$ ) binary resolved for the first time by SPHERE demonstrating the capability of the system to provide diffraction limited images for such faint objects ! 


\section{CURRENT LIMITATIONS AND FUTURE IMPROVEMENT}

\subsection{The main SPHERE limitation: The "Low wind effect"}

The so-called low wind effect [LWE] (Mickey Ears artifacts) has a significant impact on the SPHERE performance. At a level of about $20 \%$ of the time (when the wind strength in the telescope is below $1 \mathrm{~m} / \mathrm{s}$ ) some spurious artifacts appears on the PSF (located on the first airy ring).

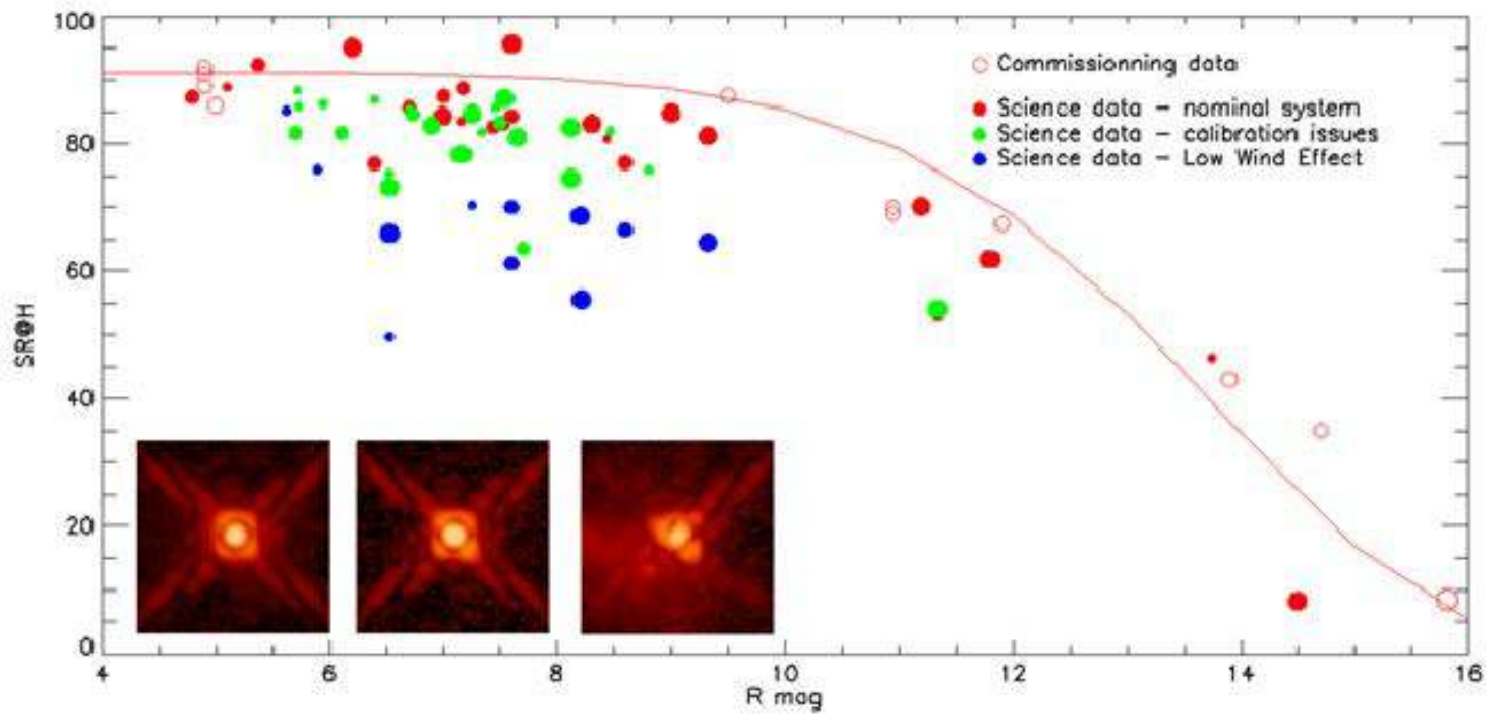

Figure 15 Comparison of commissioning data and operation data. The effects of mis-calibration is presented (problem with the reference slopes) and the $20 \%$ of low wind effect data (in blue). This highlights the need for accurate and very robust calibration procedures as well as the huge impact of the LWE. Note that during commissioning, LWE data were automatically rejected till we were sure of the problem origin (i.e. beginng of 2015)

After extensive analysis, these so-called Mickey Ears artifacts, seems due to heat exchanges between ambient air and telescope spiders, thus creating optical path differences between the two sides of the spiders. Note that the SPHERE experience has allowed demonstrating that this effect is also present on other telescopes (Gemini, Subaru, Keck ...).

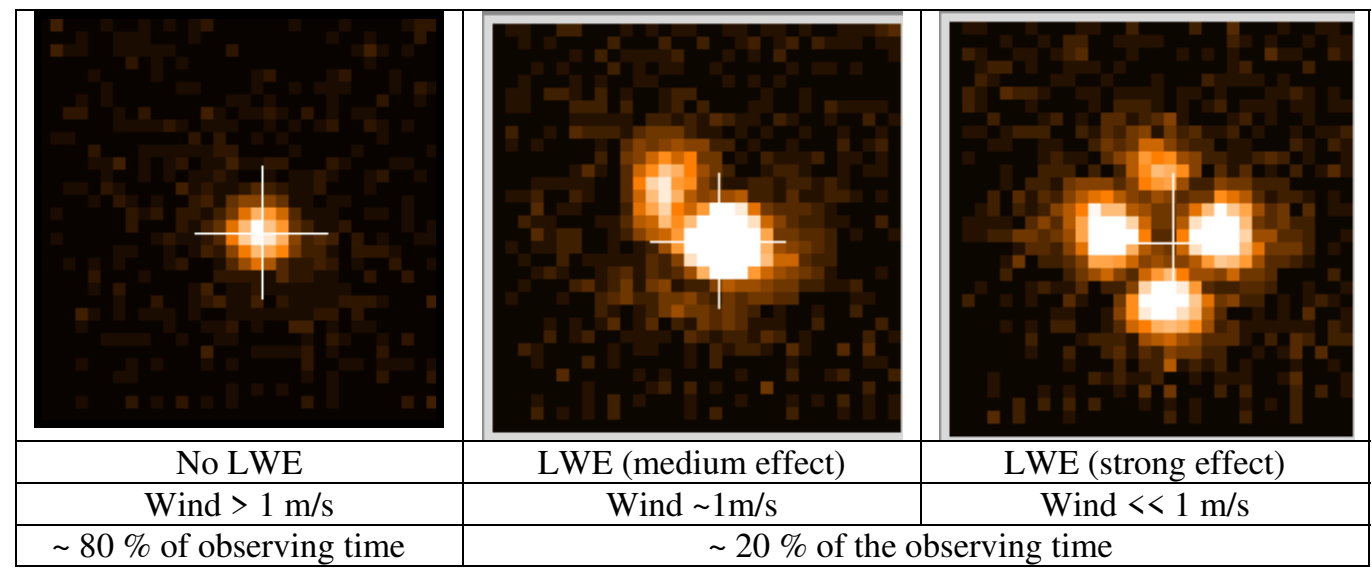

Figure 16 Example of Low wind effect PSF

A mitigation plan is being set up in collaboration between ESO and the consortium to solve the problem using DTTS signal itself, technical tests will be conducted in the first quarter of 2016.

More details on that particular effect could be found in [17]. 


\subsection{ZELDA with SPHERE}

Over the past few years, several methods have been proposed to measure and correct the residual NCPA. Of these concepts, the Zernike wavefront sensor represents a compelling solution based on phase-contrast methods because of the simplicity of its optical design for an easy implementation in current instruments and of the ease and accuracy of its reconstruction algorithm for the fast correction of small phase errors at a nanometric level.

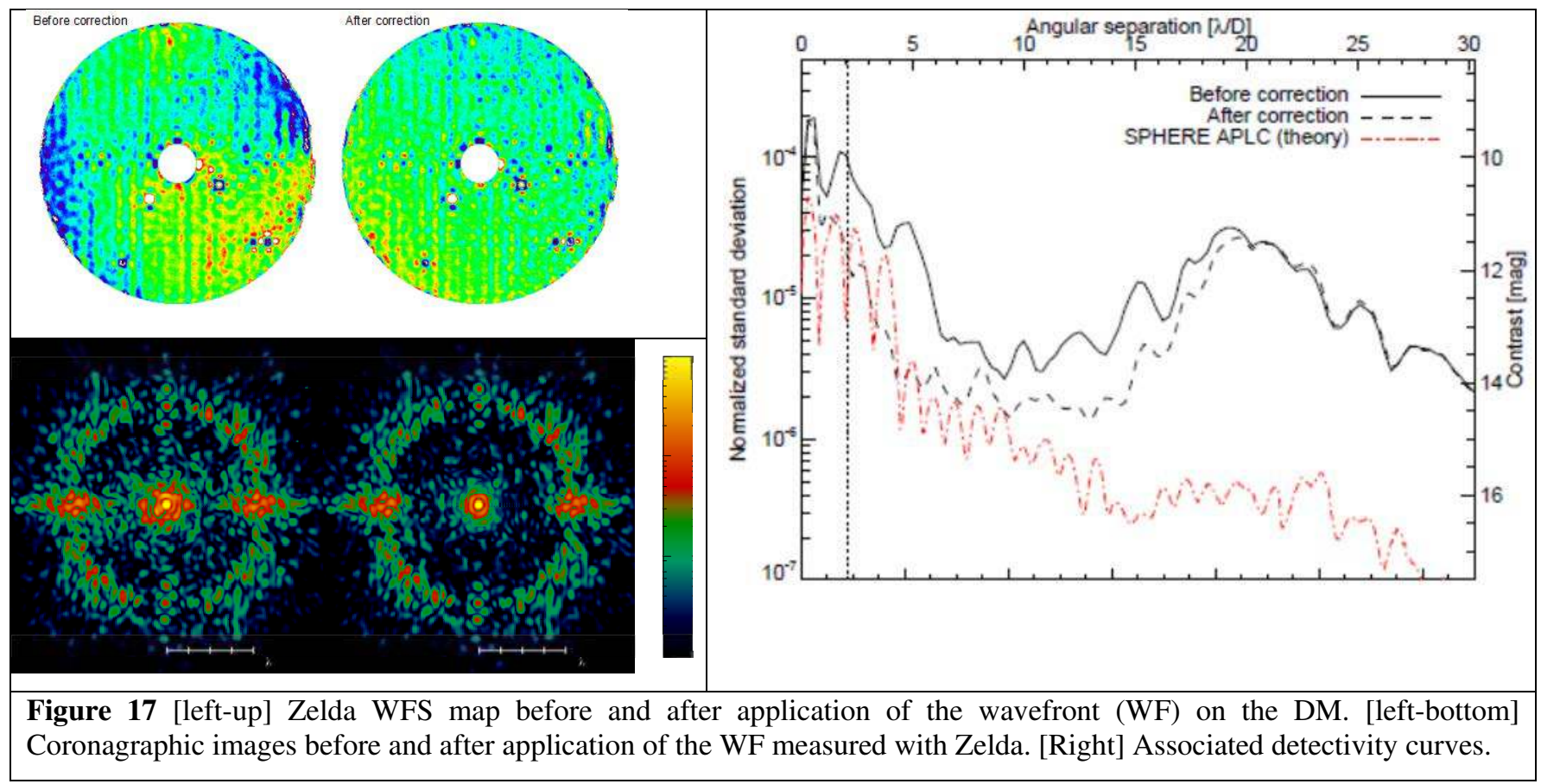

Over the past fifteen years, such a concept has been investigated in astronomical applications for multiple purposes [19], such as wavefront sensing for adaptive optics systems [20], co-phasing for alignment of segmented aperture telescopes [21], or estimation of low-order aberrations (e.g. pointing errors, focus drifts) for future missions with coronagraphic capabilities[22]. We have proposed ZELDA [23-26] as a concept based on the Zernike phase contrast applied to the measurements of quasi-static aberrations in exoplanet direct imaging instruments. Following our concept studies, we manufactured a prototype that was inserted into VLT/SPHERE in its IR coronagraph wheel during the re-integration of the instrument at Paranal in 2014. In Dec. 2015, we performed tests that led to the experimental validation of our concept [27 and this conference]. These first results show that calibration of NCPA using ZELDA can significantly reduce the residual speckle brightness in coronagraphic images for exoplanet observation, see Figure 17 We have also used ZELDA as a diagnostic tool to understand the origin of the unforeseen low-wind effect [28 and this conference], see Section 5.1.

\subsection{Dark hole with SPHERE}

The SPHERE instrument has been used as an experimental test bench to perform some validation of techniques dedicated to very high contrast. Among these techniques, the Electric Field Conjugation [EFC] [18] could be tested both on internal source and on-sky.

The EFC technique is a speckle nulling technique aiming at suppressing the light residuals coming from phase and amplitude aberrations in the optical system. This technique uses the focal plane information to retrieve the voltage modifications to be applied on the deformable mirror allowing such a nulling. For better performance (hence larger contrast values), the effect of the technic can be reduced to a given area in the focal plane.

This technique requires the calibration of the interaction matrix between the influence functions of the deformable mirror and the focal plane information. This matrix is calibrated in Fourier space, by applying to the HODM the spatial 
frequency able to generate a modification in the area of the Dark Hole. This application is made via a modification of the reference slopes in closed loop, so to ensure that the correction is compatible with an on-sky performance. Even if the calibration of this matrix is time-consuming, the calibrated matrix seems to be stable to be re-used a few month after the calibration.

The Figure 18 left shows an impressive gain of a factor 16 on the raw contrast obtained on internal source. The dark hole area is visible on the top of the AO corrected area, as a rectangular shape.

The Figure 18 right shows the on-sky performance. The reference slopes obtained at the end of the EFC internal source calibration are used to obtain this image. The gain in contrast, even if reduced by the atmospherical AO-corrected residuals, is still at a factor of 8 .
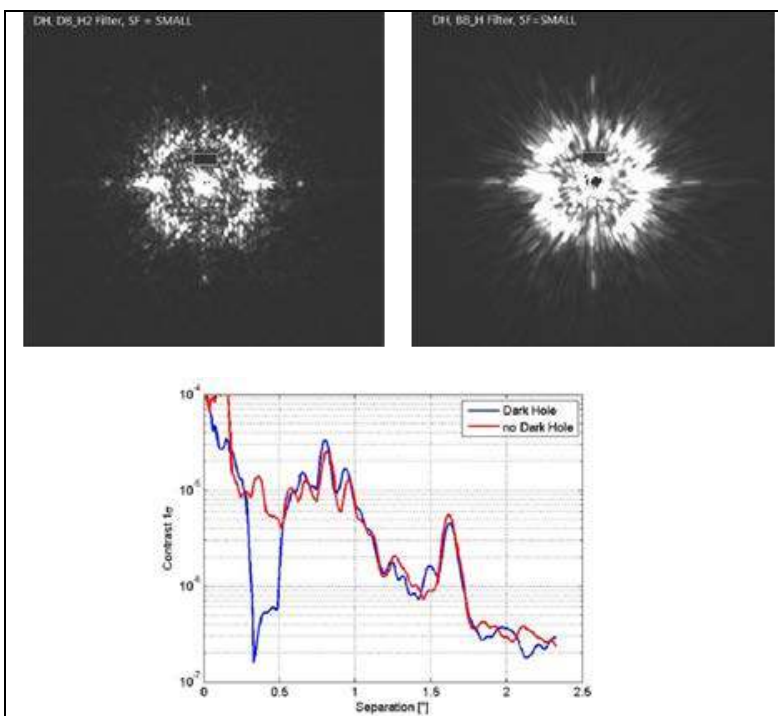

Internal source
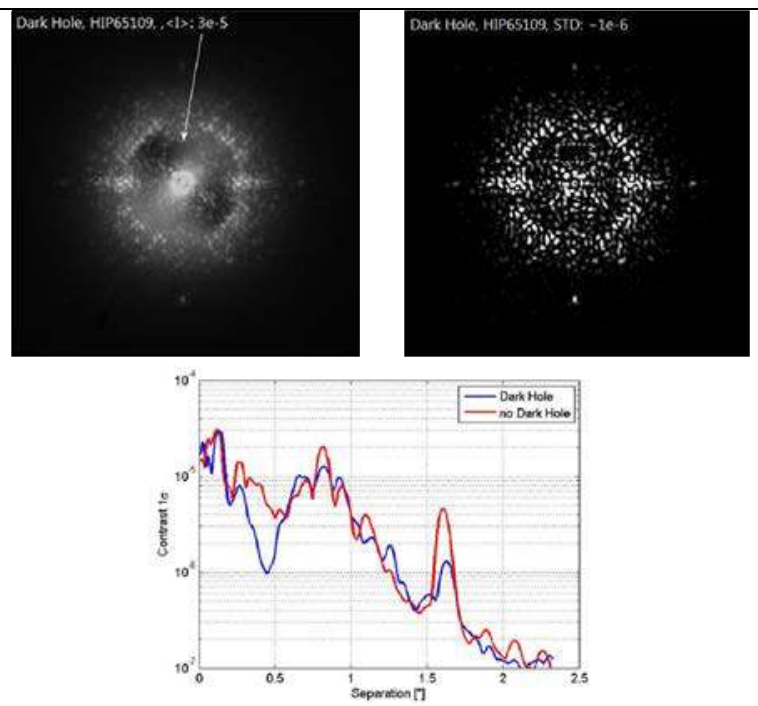

On sky data

Figure 18: result of EFC application on SPHERE instrument. Internal performance [left] shows a gain as well as on-sky performance [right] are shown.

\section{SOME UNIQUE SPHERE FEATURES ILLUSTRATED ON SCIENTIFIC IMAGES}

\subsection{Disk}

Even though SPHERE primary goal is the detection of extrasolar planet (today there is numerous candidates to be confirmed and some characterization of already detected exoplanet systems [29]), the early and more visible success of the instrument is, without any contest, is related to the disk observation. In that respect, the unique SPHERE features (large FoV in NIR, visible imaging, polarimetric capabilities in NIR and VIS) have led to exceptional data and results see ([30], [31]) 


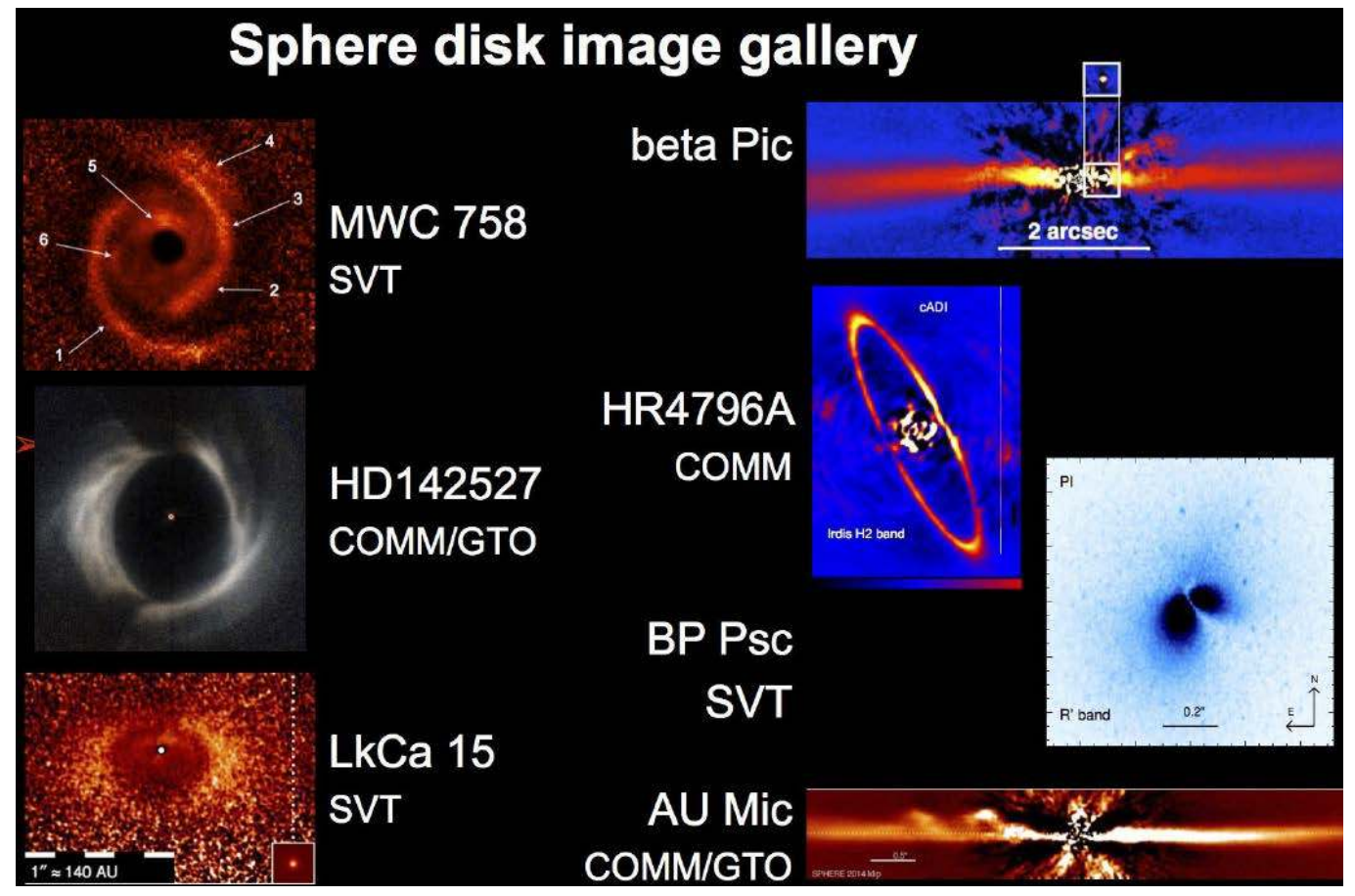

Figure 19 Non-exhaustive bestiary of disk observation with SPHERE. The unique combination ofHigh SR, stable PSF, VIS imaging, polarimetry and "NIR FoV" makes SPHERE unique for disk observations

6.2 Very high angular resolution and polarization

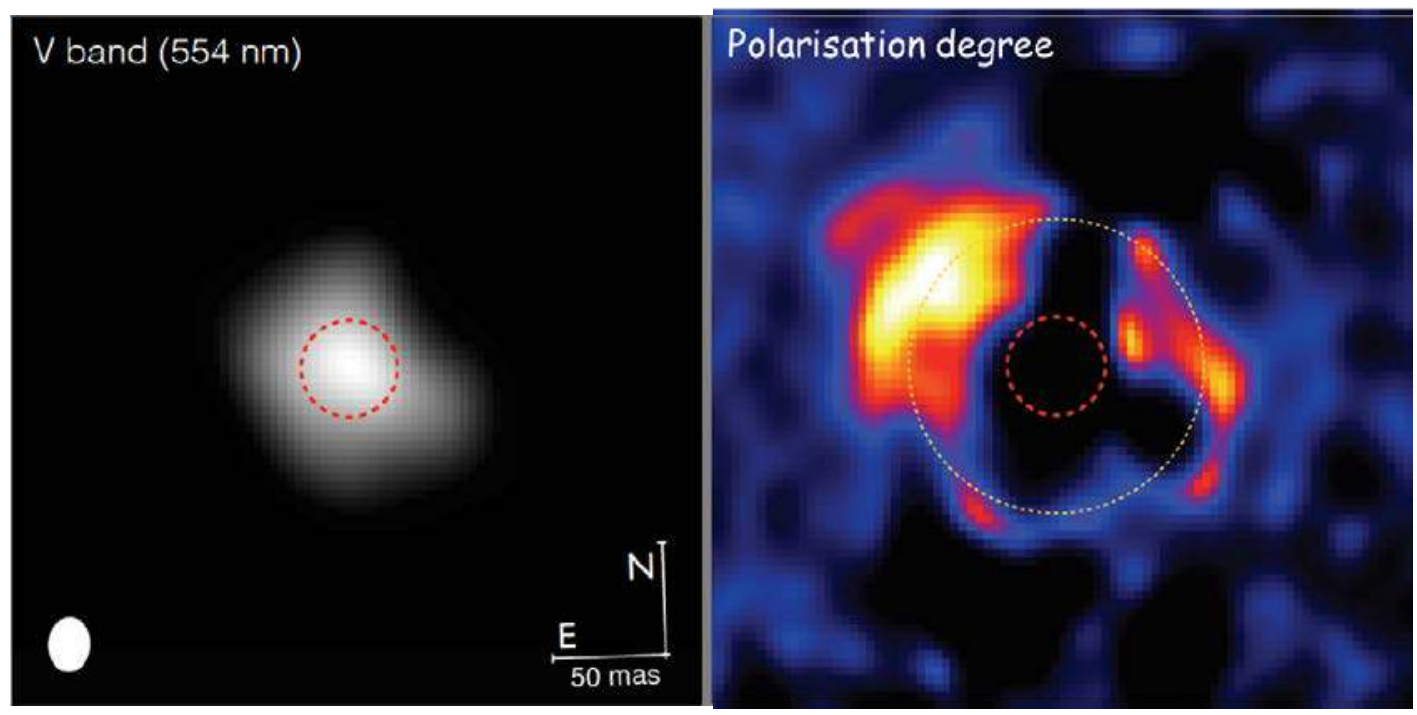

Figure 20 Betelgeuse: first "direct" image" of its photosphere. Dust within 3 stellar radii: important for mass loss? Karvella et al 2015 [32] 


\section{3 "Large" NIR FoV}
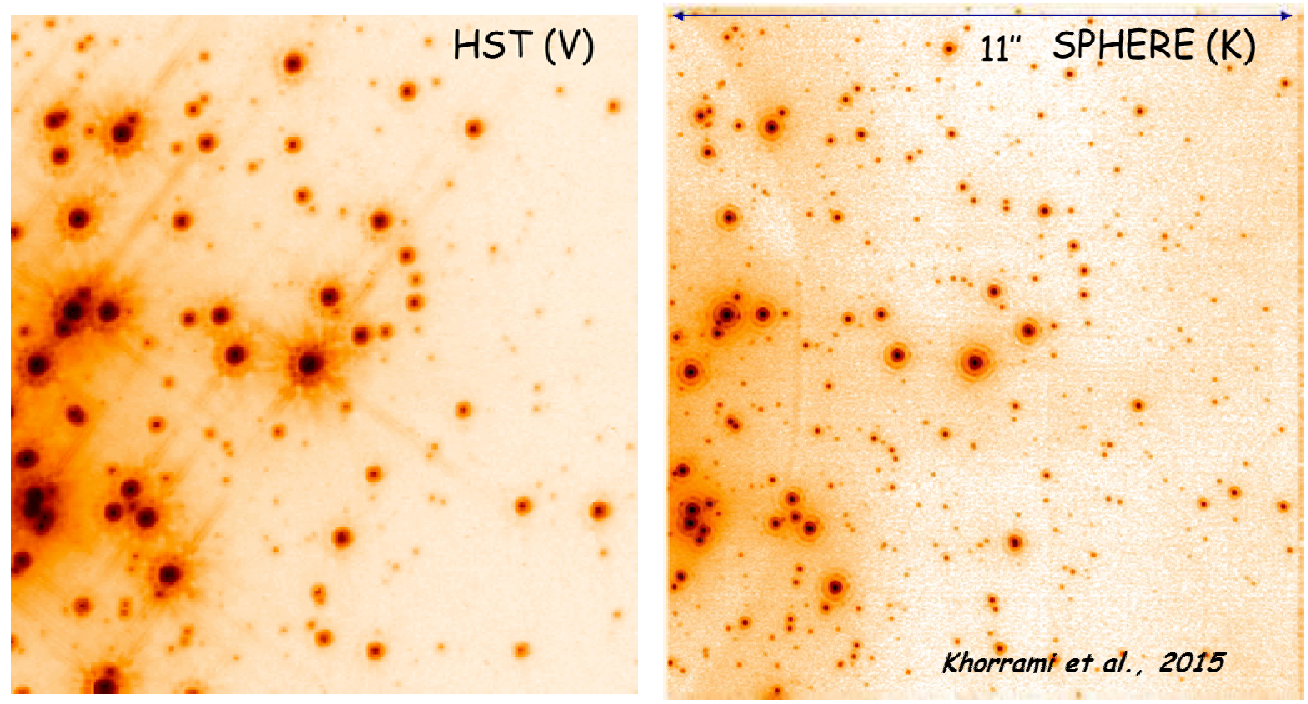

Figure 21 SPHERE Kmag down to $18:$ twice more sources detected than by the HST

\subsection{Ultimate angular resolution in the Visible}
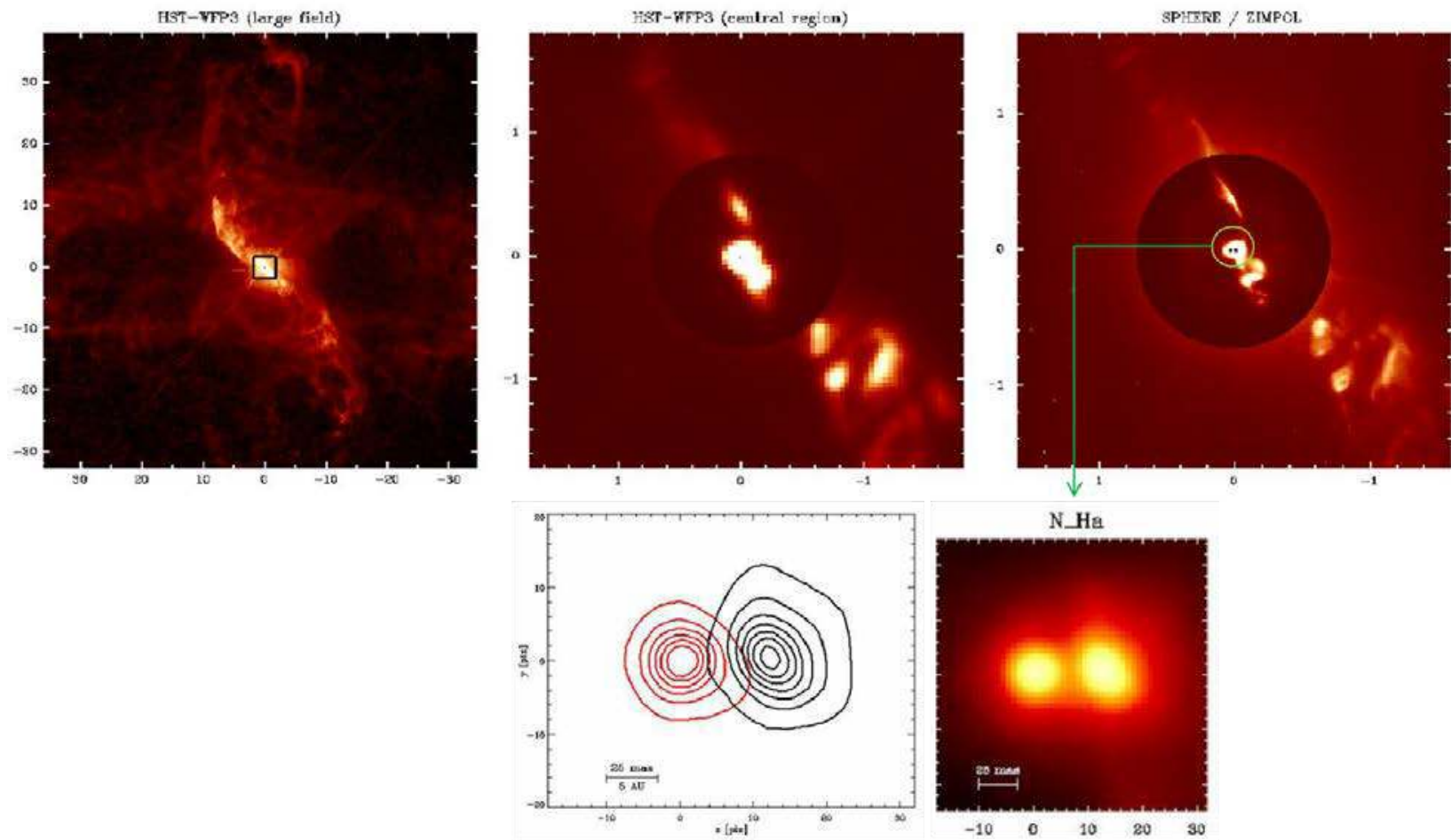

Figure 22 R-Aq observation with Zimpol. [Up-Left] : HST wide field image [Up-center] HST zoom on the central region. [Up-Right] Zimpol image at the same wavelength. [bottom] zoom on the central binary with a separation of 25 mas (i.e. $6 \mathrm{AU})$ 


\section{CONCLUSION}

SPHERE-SAXO is 12 years of project involving 12 institutes in Europe and more than 250 Full Time Equivalents. The system design has barely evolved from the early sketches (in 2002) to the final telescope implementation (2013-14). At the end, the AO system gathers state of the art components (detectors, deformable mirror and real time computer) and some of its innovative features allow having performance which has exceeded the original specifications. Even though we have experimented some problems with our high order deformable mirror, solutions have been proposed by the consortium to mitigate these effects and to be able to work with the current device. Nevertheless, DM remains, for the moment, the main risk of failure for the whole system. Back-up, long-term, solutions are now under investigation between ESO, CILAS and the SPHERE consortium to deal with this particular point.

Extensive laboratory tests have fully demonstrated the performance, the reliability and the stability of the AO loops (and of SAXO and SPHERE as a whole).The first on-sky commissioning has confirmed laboratory tests by providing unprecedented images and coronagraphic data on the VLT. The system stability has been re-demonstrated on-sky and the performance assessment, after one year of commissionning and verification period is fully coherent with the initial specifications. The main and unique features of SAXO have already been successfully tested and are now routinely used in operation. The fine tuning of the AO system as well as of the telescope control has allowed to reach the full system performance and to already produce unique science data.

In one year and an half of operation SPHERE has already generated almost 40 published paper in peer-review journals

(Nature, Science ...) and its first exoplanet discovery has been announced the $7^{\text {th }}$ of July (see

http://www.eso.org/public/news/eso1624/ and [34])

\section{ACKNOWLEDGMENT}

The authors thank the whole SPHERE consortium and ESO AO group for more than 10 years of hard and fruitful work. The work was partly funded by the European Commission under FP7 Grant Agreement No. 312430 Optical Infrared Coordination Network for Astronomy and by the French Aeorspace lab (ONERA) in the framework of the NAIADE Research Project.

\section{REFERENCES}

[1] J-L. Beuzit, D. Mouillet, C. Moutou, K. Dohlen, P. Puget, T. Fusco, and A. Boccaletti, "A planet finder instrument for the VLT," in Proceedings of IAU Colloquium 200, Direct Imaging of Exoplanets: Science \& Techniques (Cambridge University Press, 2005), pp. 317-323

[2] T. Fusco et al "High-order adaptive optics requirements for direct detection of extrasolar planets: Application to the SPHERE instrument" Optics Express, Vol. 14, Issue 17, pp. 7515-7534 (2006)

[3] J-F sauvage et al "SAXO, the eXtreme AO system of SPHERE (I) System overview and global laboratory performance", Journal of Astronomical Telescopes, Instruments, and Systems, 2016, accepted for publication

[4] J.-F; Sauvage et al "Wave-front sensor strategies for SPHERE: first on-sky results and future improvements", paper 9148-155, this proceeding

[5] K. Dohlen et al. "The infra-red dual imaging and spectrograph for SPHERE: design and performance", 7014, jul 2008, SPIE.

[6] R. Claudi et al, "The SPHERE IFS at work", Proc. SPIE 9147, Ground-based and Airborne Instrumentation for Astronomy V, 91471L (8 July 2014)

[7] R Roelfsema et al, "The ZIMPOL high contrast imaging polarimeter for SPHERE: system test results", Proc. SPIE 9147, Ground-based and Airborne Instrumentation for Astronomy V, 91473W (8 July 2014)

[8] Sauvage J.-F., Fusco T., Rousset G., Petit C., "Calibration and Pre-Compensation of Non-Common Path Aberrations for eXtreme Adaptive Optics", JOSA A, Vol. 24, Issue 8, pp. 2334-2346 (2007)

[9] Hugot et al, "Active optics methods for exoplanets direct imaging", A\&A 538, A139 (2012)

[10] T. Fusco et al, "Shack-Hartmann wavefront sensing with a low-light level CCD: from theory to practice", proc. SPIE 7015-182, Marseille (2008). 
[11] L.A.Poyneer and B. Macintosh, "Spatially filtered wave-front sensor for high-order adaptive optics", JOSA A, Vol. 21, Issue 5, pp. 810-819 (2004)

[12] T. Fusco et al "Closed-loop experimental validation of the spatially filtered Shack-Hartmann concept.", Opt Lett. 2005 Jun 1;30(11):1255-7.

[13] J.-F; Sauvage et al "Wave-front sensor strategies for SPHERE: first on-sky results and future improvements", Proc. SPIE 9148, Adaptive Optics Systems IV, 91480 O (7 August 2014)

[14] M. Nicolle, T. Fusco, G. Rousset and V. Michau, "Improvement of Shack-Hartmann wave-front sensor measurement for extreme adaptive optics", Optics Letters, Vol. 29, Issue 23, pp. 2743-2745 (2004)

[15] C. Petit et al "SPHERE eXtreme AO control scheme: final performance assessment and on sky validation of the first auto-tuned LQG based operational system", Proc. SPIE 9148, Adaptive Optics Systems IV, 914800 (7 August 2014)

[16]P. Baudoz et al, "The differential tip-tilt sensor of SPHERE", Proc. SPIE 7735, Ground-based and Airborne Instrumentation for Astronomy III, 77355B (21 July 2010)

[17] G. Montagnier, T. Fusco, J. -L. Beuzit, D. Mouillet, J. Charton, L. Jocou, Pupil stabilization for SPHERE's extreme AO and high performance coronagraph system, Opt.Express 15:15293-15307,2007Low Wind Effect, the main limitation of the SPHERE instrument, Sauvage Jean-François, Fusco Thierry et al, This conference 2015.

[18] Laboratory test of application of Electric Field Conjugation image-sharpening to ground-based adaptive optics, Thomas, S. J.; Give'On, A. A., 2010 Lyot Conference

[19] Dohlen, K., \Phase masks in astronomy: From the Mach-Zehnder interferometer to coronagraphs," in [EAS Publications Series], Aime, C. and Soummer, R., eds., 12, $33\{44$ (2004).

[20] Bloemhof, E. E. and Wallace, J. K., \Phase contrast techniques for wavefront sensing and calibration in adaptive optics," in [SPIE], 5169, 309\{320 (Dec. 2003).

[21] Vigan, A., Dohlen, K., and Mazzanti, S., IOn-sky multiwavelength phasing of segmented telescopes with the Zernike phase contrast sensor," Applied Optics 50, 2708 (June 2011).

[22]Zhao, F., IWFIRST-AFTA coronagraph instrument overview," in [SPIE], 9143, 0 (Aug. 2014).

[23] N'Diaye, M., Dohlen, K., Fusco, T., and Paul, B., ICalibration of quasi-static aberrations in exoplanet directimaging instruments with a Zernike phase-mask sensor," Astron. \& Astrophys. 555, A94 (July 2013).

[24] N'Diaye, M., Dohlen, K., Fusco, T., El Hadi, K., Soummer, R., Cuevas, S., Zerrad, M., and Ferrari, M., ILab results of the circular phase mask concepts for high-contrast imaging of exoplanets," in [SPIE], 8450 (Sept. 2012).

[25] Dohlen, K., Madec, F., N'Diaye, M., Paul, B., Fusco, T., Jolivet, A., Luo, D., Yatcheva, L., Sauvage, J.-F., Mugnier, L., and Ferrari, M., Lab demonstration of the Zernike phase mask near-coronagraph quasi static aberrations sensor, ZELDA," in [Proceedings of the Third AO4ELT Conference], Esposito, S. and Fini, L., eds., 86 (Dec. 2013).

[26] N'Diaye, M., Dohlen, K., Caillat, A., Costille, A., Fusco, T., Jolivet, A., Madec, F., Mugnier, L., Paul, B., Sauvage, J.-F., Soummer, R., Vigan, A., and Wallace, J. K., IDesign optimization and lab demonstration of ZELDA: a Zernike sensor for near-coronagraph quasi-static measurements," in [SPIE], 9148, 5 (Aug. 2014).

[27] N'Diaye, M., Vigan, A., Dohlen, K., Sauvage, J.-F., Caillat, A., Costille, A., Girard, J. H. V., Beuzit, J.-L., Fusco, T., Blanchard, P., Le Merrer, J., Le Mignant, D., Madec, F., Moreaux, G., Mouillet, D., Puget, P., and Zins, G., ICalibration of quasi-static aberrations in exoplanet direct-imaging instruments with a Zernike phasemask sensor. II. Concept validation with ZELDA on VLT/SPHERE," accepted A\&A, ArXiv e-prints (June 2016).

[28] Sauvage, J. F., Fusco, T., Andres, G., Wizinowich, P., O'Neal, J., N'Diaye, M., Vigan, A., Girard, J., Lesur, G., Mouillet, D., Beuzit, J.-L., Kasper, M., Le Louarn, M., Milli, J., Dohlen, K., Neichel, B., Bourget, P., Hagenauer, P., and Mawet, D., LLow Wind E ect, the main limitation of the SPHERE instrument," in [Proceedings of the Fourth AO4ELT Conference], Esposito, S. and Fini, L., eds. (2016).

[29] M Bonnefoy, A Zurlo, JL Baudino, P Lucas, D Mesa, A-L Maire, A Vigan, R Galicher, D Homeier, F Marocco, R Gratton, G Chauvin, F Allard, S Desidera, M Kasper, C Moutou, A-M Lagrange, J Antichi, A Baruffolo, J 
Baudrand, J-L Beuzit, A Boccaletti, F Cantalloube, M Carbillet, J Charton, RU Claudi, A Costille, K Dohlen, C Dominik, D Fantinel, P Feautrier, M Feldt, T Fusco, P Gigan, JH Girard, L Gluck, C Gry, T Henning, M Janson, M Langlois, F Madec, Y Magnard, D Maurel, D Mawet, MR Meyer, J Milli, O Moeller-Nilsson, D Mouillet, A Pavlov, D Perret, P Pujet, SP Quanz, S Rochat, G Rousset, A Roux, B Salasnich, G Salter, J-F Sauvage, HM Schmid, A Sevin, C Soenke, E Stadler, M Turatto, S Udry, F Vakili, Z Wahhaj, F Wildi, First light of the VLT planet finder SPHERE-IV. Physical and chemical properties of the planets around HR8799, A\&A, 587, 2016

[30] C Perrot, A Boccaletti, E Pantin, J-C Augereau, A-M Lagrange, R Galicher, A-L Maire, J Mazoyer, J Milli, G Rousset, R Gratton, M Bonnefoy, W Brandner, E Buenzli, M Langlois, J Lannier, D Mesa, S Peretti, G Salter, E Sissa, G Chauvin, S Desidera, M Feldt, A Vigan, E Di Folco, A Dutrey, J Péricaud, P Baudoz, M Benisty, J De Boer, A Garufi, JH Girard, F Menard, J Olofsson, SP Quanz, D Mouillet, V Christiaens, S Casassus, J-L Beuzit, P Blanchard, M Carle, T Fusco, E Giro, N Hubin, D Maurel, O Moeller-Nilsson, A Sevin, L Weber, Discovery of concentric broken rings at sub-arcsec separations in the HD 141569A gas-rich, debris disk with VLT/SPHERE, A\&A, 590, 2016

[31] Anthony Boccaletti, Christian Thalmann, Anne-Marie Lagrange, Markus Janson, Jean-Charles Augereau, Glenn Schneider, Julien Milli, Carol Grady, John Debes, Maud Langlois, David Mouillet, Thomas Henning, Carsten Dominik, Anne-Lise Maire, Jean-Luc Beuzit, Joseph Carson, Kjetil Dohlen, Natalia Engler, Markus Feldt, Thierry Fusco, Christian Ginski, Julien H Girard, Dean Hines, Markus Kasper, Dimitri Mawet, François Ménard, Michael R Meyer, Claire Moutou, Johan Olofsson, Timothy Rodigas, Jean-Francois Sauvage, Joshua Schlieder, Hans Martin Schmid, Massimo Turatto, Stephane Udry, Farrokh Vakili, Arthur Vigan, Zahed Wahhaj, John Wisniewski, Fast-moving features in the debris disk around AU Microscopii, Nature, 526, 7572, 2015

[32] Kervella, P.; Lagadec, E.; Montargès, M.; Ridgway, S. T.; Chiavassa, A.; Haubois, X.; Schmid, H. -M.; Langlois, M.; Gallenne, A.; Perrin, G., The close circumstellar environment of Betelgeuse. III. SPHERE/ZIMPOL imaging polarimetry in the visible, A\&A, 585, 2016

[33]Z. Khorrami, T. Lanz, F. Vakili, E. Lagadec, M. Langlois, W. Brandner, O. Chesneau, M. R. Meyer, M. Carbillet, L. Abe, D. Mouillet, JL. Beuzit, A. Boccaletti, C. Perrot, C. Thalmann, H.-M. Schmid, A. Pavlov, A. Costille, K. Dohlen, D. Le Mignant, C. Petit, J.F. Sauvage, VLT/SPHERE deep insight of NGC 3603's core: Segregation or confusion?, A\&A, 588, 2016

[34] Kevin Wagner, Dániel Apai, Markus Kasper, Kaitlin Kratter, Melissa McClure, Massimo Robberto, JeanLuc Beuzit, Direct imaging discovery of a Jovian exoplanet within a triple-star system, Science 07 Jul 2016, DOI: $10.1126 /$ science.aaf967 